ECCOMAS

\section{Proceedia}

COMPDYN 2021

$8^{\text {th }}$ ECCOMAS Thematic Conference on Computational Methods in Structural Dynamics and Earthquake Engineering

M. Papadrakakis, M. Fragiadakis (eds.) Streamed from Athens, Greece, 28 - 30 June 2021

\title{
APPLICATION TO AN URBAN BRIDGE STOCK OF A PRIORITIZATION PROCEDURE BASED ON SEISMIC ASSESSMENT COMPARED WITH THE NOVEL ITALIAN GUIDELINES
}

\author{
E. Saler ${ }^{1,3}$, V. Pernechele ${ }^{2}$, G. Tecchio ${ }^{3}$ and F. da Porto ${ }^{3}$ \\ ${ }^{1}$ Dept. of Civil, Environmental and Mechanical Engineering, University of Trento \\ Via Mesiano 77, Trento, Italy \\ e-mail: elisa.saler@unitn.it \\ ${ }^{2}$ Institute for Construction Technologies - National Research Council (ITC-CNR) \\ Corso Stati Uniti 4, 35127 Padova, Italy \\ e-mail: pernechele@itc.cnr.it \\ ${ }^{3}$ Dept. of Geosciences, University of Padova \\ Via Gradenigo 6, 35131 Padova, Italy \\ e-mail: \{giovanni.tecchio,francesca.daporto\}@unipd.it
}

\begin{abstract}
The challenge of large-stock maintenance with limited budget is faced by administrations in charge which need tools for management and intervention prioritization. This need is especially perceived for bridges which are strategic structures and represent crucial elements in roadway networks. Since 2016, a combined prioritization procedure has been applied to the bridge stock of the Municipality of Padova (Italy) to assess both the condition state, accounting for degradation, and the seismic vulnerability. The proposed methodology evaluates the level of degradation through visual inspections and type-specific forms for the defect survey, while the seismic vulnerability is assessed by means of fragility curves previously obtained by the authors for typical Italian bridges. This contribution focuses on the application of the seismic-based prioritization. The identification of the bridge typologies which appeared to be more seismically fragile is presented, comparing this aspect with results from the recently issued novel Italian Guidelines for the management of existing bridges.
\end{abstract}

Keywords: Bridge stock, bridge degradation, condition state assessment, bridge seismic vulnerability, prioritization. 


\section{INTRODUCTION}

Roadway networks represent strategic infrastructures for the civil protection system, since they are fundamental for post-event emergency management. Thus, disaster risk mitigation requires also to improve the reliability of infrastructure networks, especially their most vulnerable elements, such as bridges.

Most of Italian bridges were built before 1980 [1], with no seismic provisions, since the seismic classification of large part of the country was completed only by the early 2000 s.

Focusing on seismic vulnerability of bridge assets, fragility curves represent an effective tool describe the susceptibility of a structure to seismic damage. They expresses the probability of exceedance a certain performance level (or damage state [2]) for a given value of intensity measure, for instance the peak ground acceleration (PGA).

An extensive review of the fragility assessment approach in the literature was provided by Muntasir Billah and Shahria Alam [3]. Analytical models are widely used to derive fragility functions, as they can be applied to general taxonomies of structure. One of the most acknowledged analytical approach is the derivation of fragility curves through non-linear time history analysis (NLTHA) applied to mechanical models (e.g., [4-6]), also considering the effect of degradation on structural performance [7]. Mechanics-based vulnerability assessment of bridges can be also carried out through simplified procedures, such as Displacement-Based approaches [8,9] or limit analysis applied to arch bridges [10,11].

A number of authors have focused on seismic vulnerability assessment of typical bridges of a particular region or country, so as to better catch the local bridge taxonomy [12-14].

Moreover, bridges are particularly subjected to degradation, due to their exposure to environmental agents, as well as other natural hazards (e.g., flood, scour, and landslide) [15]. In addition, some structures, such as orthotropic steel deck bridges, might be subjected to fatigue phenomena, even if recently built [16].

In this framework, structural health monitoring (SHM) systems can be applied to the most critical or strategic structures, to efficiently collect information on structural integrity and durability [17].

Nevertheless, the maintenance and retrofit of large bridge stock is a challenging theme for administrations in charge, that need to allocate limited funds.

A series of scientific-based bridge management systems (BMS) have been proposed since the early 90 s [18-21]. In addition, prioritization procedures are commonly required when infrastructure maintenance has been neglected for a long time, leading to the need of an extensive restoration and retrofit campaign which must be first addressed to the most critical structures [20]. Various prioritization approaches have been proposed by researchers, such as, among the others [22-24].

More recently, research has started to focus on combined prioritization approaches, which include seismic vulnerability and degradation. In this framework, in 2020, novel guidelines for existing bridges were issued in Italy [25]. They proposed a novel simplified and multi-level procedure to prioritize further verifications and interventions on bridges part of a stock, based on a multi-risk approach, which considers structural safety accounting for degradation, as well as seismic, hydraulic, and hydrogeological risks. The main limitation of the proposed procedure is that it groups bridges in attention classes, but it has not the purpose of a detailed ranking by rating each structure.

This paper discusses a priority ranking procedure which evaluates both degradation effects and seismic vulnerability (the former through visual inspections and defect survey, and the latter by using fragility curves from the literature), as described by the authors in Saler et al. [26]. The estimate of degradation effects was carried out according to a simplified procedure 
available in the literature [27], whereas the proposed seismic assessment approach was devised so as to be consistent with it.

Since 2016, the prioritization approach has been applied to an urban bridge stock consisting of more than 160 bridges. The analyzed urban area, whose specific features are described in the paper, is the Municipality of Padova, in North-East Italy.

This contribution is focused on seismic evaluation, for which a comparison with the procedure proposed by Italian Guidelines is provided [25].

\section{THE BRIDGE INVENTORY OF PADOVA, ITALY}

The current section presents the bridge stock managed by the Municipality of Padova (i.e., comprised of structures for which the local administration is directly in charge, as well as bridges which overcoming roads of the municipality network). Since 2016, an extensive campaign of visual inspection and survey has been carried out, allowing a detailed taxonomy of the stock to be devised.

The bridge inventory of Padova consists of 162 bridges of various types, whose characteristics reflect the topography and urbanization of the area. The city of Padova is very ancient, the first urban settlement was founded in the XII century BC, and surrounded by rivers and artificial canals, useful in the past for both transportation and defense purposes. A certain number of bridges from the Roman and Medieval ages are still operational. Railway network passes across the historical center (HC), where the railway station is located. Thus, a series of bridges were built to overcome railways tracks.

Figure 1 shows some examples of type of bridges of the Padova inventory.

Aggregated distributions of structural and typological characteristics of the bridge inventory are reported in Figure 2, unbundling data related to the $\mathrm{HC}$, so as to highlight its particular features. The related meaning of symbols is reported in Table 1.

Most bridges in the inventory are road bridges, followed by pedestrian bridges, most of which were built in the last 20 years, to enhance pedestrian safety against the increasing traffic volume. The construction age of the asset is quite heterogeneous, with a peak of structures built before 1920, including most of the masonry arched bridges in the historical center. Then, a second peak period of construction is found between 1960 and 1980, due to the expansion outside the historical center of both urbanized and productive areas. Finally, the last period of enlargement of the urban network have occurred in the last 20 years, corresponding to the construction of the most recent part of the suburban beltway, carried out in the early 2000, as well as to the realization of most pedestrian bridges, as aforementioned.

Most bridges in the HC are masonry or r.c. structures. Several cases were surveyed in which an r.c. deck was built next to a masonry arch to allow the carriageway to be widened. Out of the $\mathrm{HC}$, the majority of bridges (almost 60\%) has r.c. or prestressed r.c. deck, and the prevalence increases to $70 \%$ if only road bridges are considered.

While representing a minority part of the stock, other types of bridges (i.e., with masonry or steel deck) represents a not negligible portion of an urban asset like the Padova inventory. On the contrary, datasets referring to highway networks $[13,14]$ reported bridge types other than r.c. structures to be rarer than in the analyzed case.

Focusing on static scheme, in the HC most bridges are arched (all of masonry structures as well as a number of r.c. arch) or girders, either simply supported or continuous.

Outside the HC the distribution of static schemes appears more heterogeneous, with a peak corresponding to simply supported girders.

In the Padova inventory there are mainly single-span bridges, with also a significant set of structures with two or three spans. A greater number of spans is rare, and mainly located out of the historical center. 


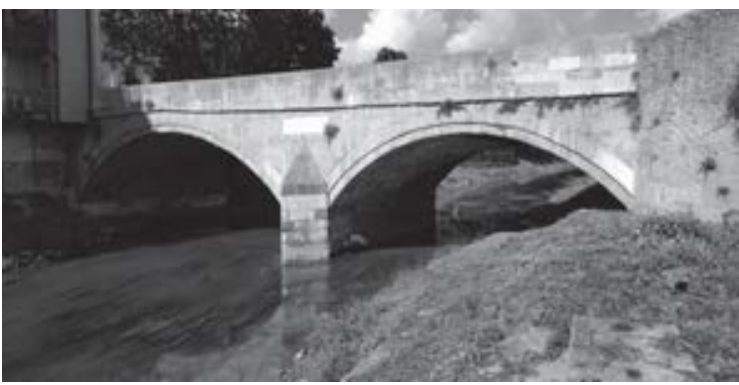

a)

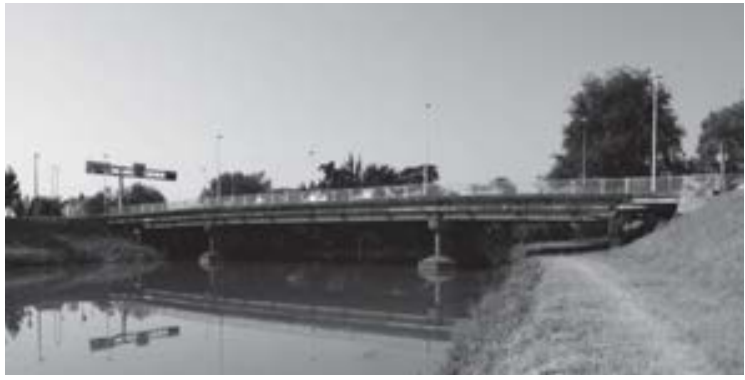

c)

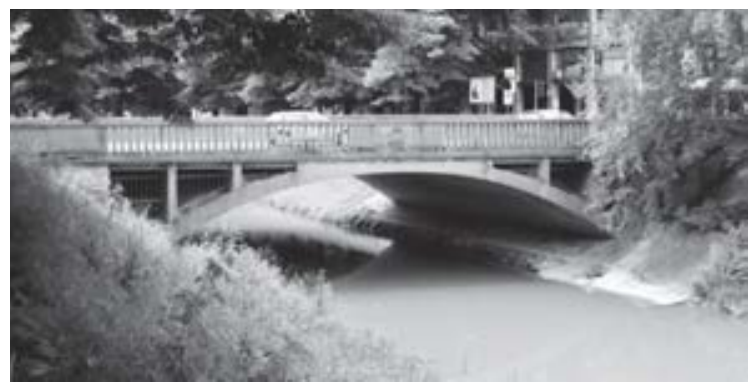

b)

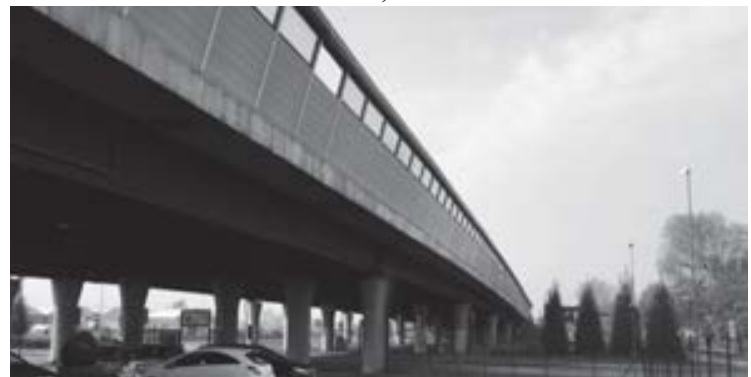

d)

Figure 1: Examples of types of bridges of Padova inventory: a) masonry arch; b) r.c. arch; c) r.c. girder deck; and d) mixed steel-r.c. girder deck.

Small $(<20 \mathrm{~m})$ to medium $(20-40 \mathrm{~m})$ bridges are mainly found in the urban area, also due to its flat topography and high population density. Spans tend to be on average shorter in the HC, with a mode in the range 10-20 meters, while outside the HC the span length mode is in the range 20-30 m (steel and precast r.c. technologies allowed longer spans to be realized). An exception inside the $\mathrm{HC}$ is due to the presence of the railway station and tracks, which require greater span length, up to $50 \mathrm{~m}$, to be properly overcome.

Generally, reinforced concrete appears to be the most used material for substructures, although masonry was used for ancient structures. In the historical center is therefore more common to find masonry abutments. Lastly the distribution of height and type of piers is provided (obviously referring to only multi span bridges - 24 in the HC, 50 out of HC). Pier height is mainly shorter than 8 meters, due to the flat topography of the area. Thus, squat configuration is more common for wall piers, which represent the most common type in the HC. Outside the $\mathrm{HC}$ also frame piers are fairly common, since this type is more suitable for structures with greater transverse width (i.e., with larger carriageways).

Nevertheless, the following sections are focused only on bridge types which are more susceptible to seismic damage (see the following Table 2), excluding light and flexible structures which are negligibly affected by seismic actions (i.e., steel arch bridges and cabled structures).

$\begin{array}{clcl}D C & \text { Reinforced concrete (r.c.) deck } & R E T-S I M & \text { Reticular-simply supported } \\ D P C & \text { Prestressed r.c. deck } & C B L & \text { Stayed cable or suspended bridge } \\ D M & \text { Masonry deck } & A M & \text { Masonry abutment } \\ D S & \text { Steel deck } & A C & \text { R.c. abutment } \\ D C S & \text { Mixed steel-r.c. deck } & P S C & \text { Circular single pier } \\ A R C H & \text { Arch } & P W & \text { Wall pier } \\ S I M & \text { Simply supported } & P F 2 & \text { Two-column frame pier } \\ G E R & \text { Gerber scheme } & P F M & \text { Multi-column frame pier } \\ C O N T & \text { Continuous spans } & & \end{array}$

Table 1: List of symbols. 


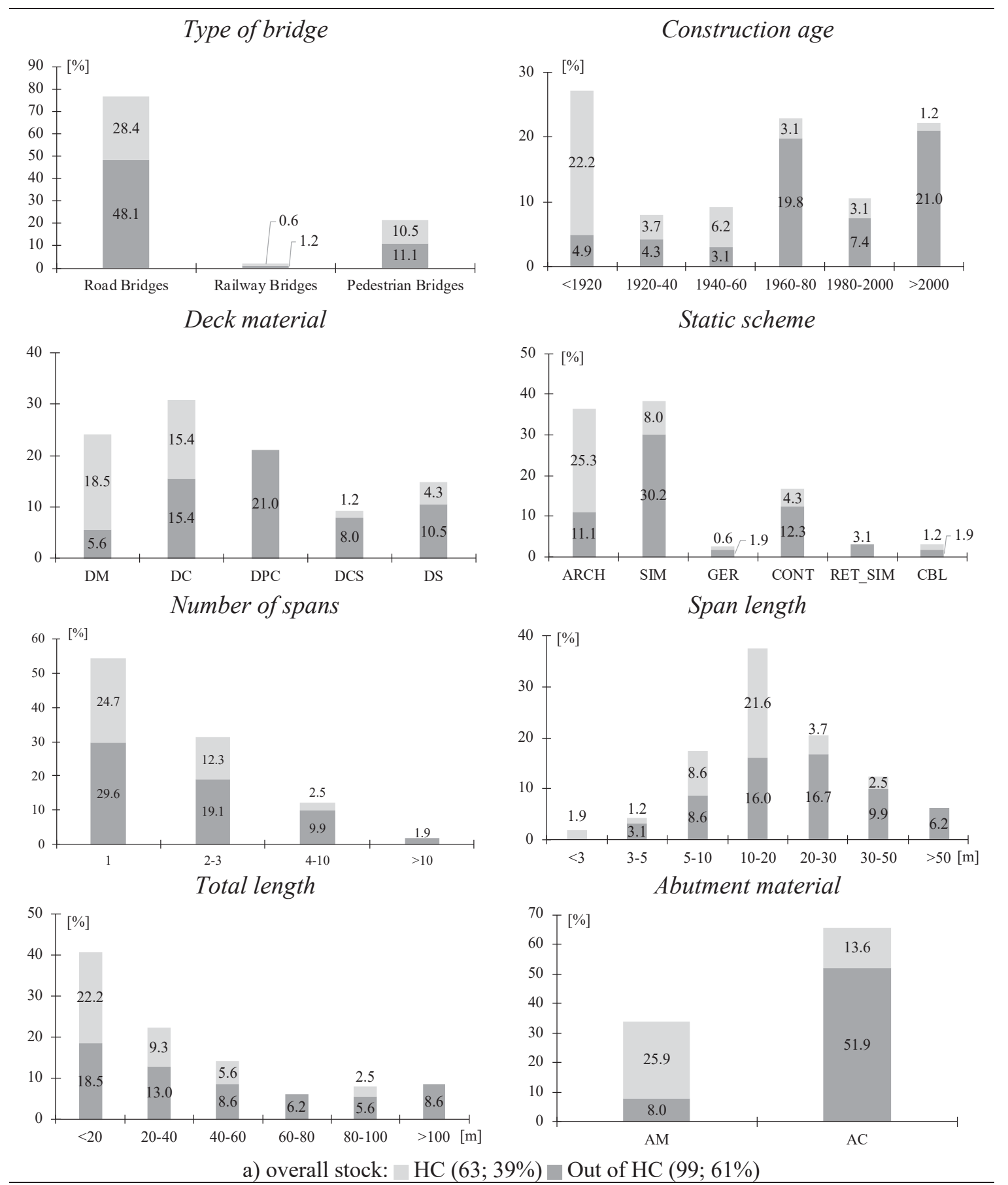

a) overall stock: $\mathrm{HC}(63 ; 39 \%)$ Out of $\mathrm{HC}(99 ; 61 \%)$

Pier type

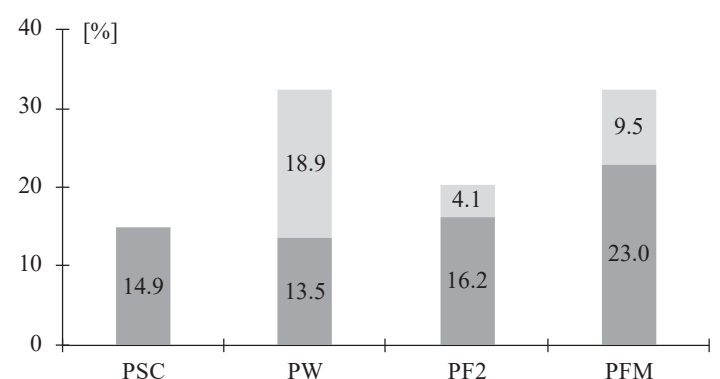

Pier height

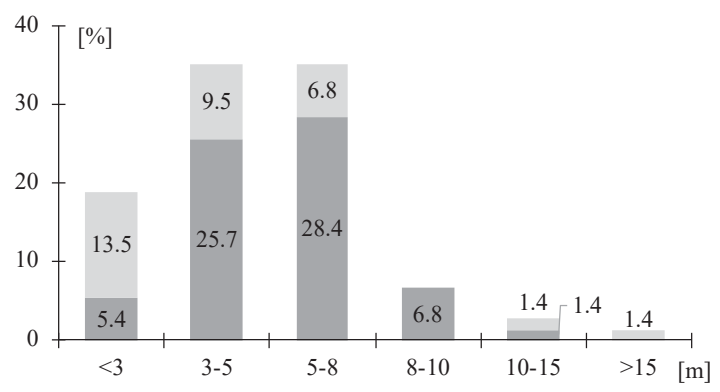

b) multi-span bridges: $\mathrm{HC}(24 ; 32 \%)$ Out of $\mathrm{HC}(50 ; 68 \%)$

Figure 2: Typological characteristics of bridges of Padova inventory: a) overall stock; b) multi-span bridges. 


\section{SEISMIC-BASED RANKING PROCEDURE}

The effects on a structure of both degradation and earthquake depends on its type and on structural members which determine how the structure responses. For instance, some components are more affected by degradation (e.g., Gerber saddles). The identification of bridge macro-classes, based on construction material and static scheme, is reported in Table 2, while Table 3 lists the structural components mainly involved in the structural response and the related mechanisms which were considered in the vulnerability assessment.

The evaluation of seismic vulnerability was carried out through fragility curves previously derived by the authors $[8,11]$, defined for ranges of geometric and structural parameters, for example different fragility curves are assigned for segmental and circular arches. Both Life Safety and Damage limit states (LSLS and DLS, respectively) were considered.

\begin{tabular}{cc}
\hline Structural Material & Static Scheme \\
\hline Masonry & Arch \\
\hline \multirow{2}{*}{ Reinforced concrete } & Arch \\
& Girder \\
\hline \multirow{2}{*}{ Steel } & Girder \\
& Reticular \\
\hline
\end{tabular}

Table 2: Macro-classes of bridges based on construction material and static scheme.

\begin{tabular}{ll}
\hline Element & \multicolumn{2}{l}{ Collapse mechanisms } \\
\hline \multicolumn{2}{l}{ Single span masonry arch } \\
\hline Arch & Longitudinal mechanism \\
Spandrel wall & Spandrel wall overturning \\
\hline Multi span masonry arch & \\
\hline Arch - Pier & Longitudinal mechanism \\
Arch - Pier & Transverse mechanism \\
Spander wall & Spandrel wall overturning \\
\hline Single span reinforced concrete/steel bridge \\
\hline Support & Support failure \\
Support & Loss of support of deck \\
Abutment & Shear/Sliding \\
Abutment & Flexural mechanism \\
\hline Multi span reinforced concrete/steel bridge \\
\hline Support & Support failure \\
Support & Loss of support of deck \\
Abutment & Shear/Sliding \\
Abutment & Flexural mechanism \\
Pier & Shear \\
Pier & Flexural mechanism \\
\hline Reinforced concrete arch \\
\hline Support & Support failure \\
Support & Loss of support of deck \\
Abutment & Shear/Sliding \\
Abutment & Flexural mechanism \\
Arch & Longitudinal mechanism \\
\hline
\end{tabular}

Table 3: Structural components and relative seismic mechanism for bridge macro-classes. 
The probability of exceeding each limit state was processed as described in detail in Saler et al. [26], obtaining a rating value (Total Sufficiency Rating - TSR [26,27]) for each bridge, ranging from 1 (highest vulnerability) to 100 (lowest vulnerability). Seismic classes were defined on the basis of seismic rating, as reported in Table 4, which as well provides an interpretation of seismic classes according to the urgency of intervention. This was defined according to a recommendation [28] of the Italian Department of Civil Protection on how to use results of seismic verifications of relevant and strategic structures, made mandatory by Italian Code OPCM 3274/2003 [29].

\begin{tabular}{c|ccc} 
& Seismic classes & TSR $_{\text {seismic }}$ & Urgency of intervention \\
\hline \multirow{2}{*}{} & $\mathrm{A}_{\mathrm{S}}$ & $76-100$ & Long-term intervention - no need for planning \\
& $\mathrm{B}_{\mathrm{S}}$ & $51-75$ & Mid-term planning of intervention \\
& $\mathrm{C}_{\mathrm{S}}$ & $26-50$ & Short-term planning of intervention \\
& $\mathrm{D}_{\mathrm{S}}$ & $1-25$ & Immediate planning of intervention \\
\hline
\end{tabular}

Table 4: Seismic classes and relative urgency of intervention.

As an example, the calculation of the seismic rating value for one representative bridge is reported as follows. The case study is a precast r.c. girder bridge; it has three spans, each one simply supported (on simple neoprene bearing pads). It has r.c. abutments and squat r.c. wall piers.

The failure mechanisms which were included in the evaluation were both shear and flexural failure of piers and abutments, as well as the failure of supports. For each mechanism the probability of exceeding either LSLS or DLS was calculated by means of fragility curves, based on the expected peak ground acceleration (PGA) for the site of the structure. Then, for each structural element, the probability of exceeding each LS was assumed to be the maximum probability among the potential mechanisms for the specific bridge component.

Then, the exceedance probability associated to the global response of the bridge was computed by combining the probability of its components, through the upper-bound approach proposed by Choi et al. [4]. The upper-bound, which is more conservative and therefore used to calculate the global exceedance probability, is based on the assumption of statistically independent structural components. The values of exceedance probability for the presented case study are reported in Table 5.

For each limit state, the exceedance probability was associated to a Condition Factor (CF) $[26,27]$, assigned as illustrated in Figure 3, which was then used to calculate the above-mentioned seismic Total Sufficiency Rating $\left(\mathrm{TSR}_{\text {seismic}}\right.$ ), including corrective coefficients to take into account the importance of the bridge in the network [27]. The final rating of the structure is obtained as the minimum TSR between the rates calculated for LSLS and DLS; the seismic class of the structure is then assigned accordingly (Table 6).

A seismic class Ds was therefore assigned to the presented case study, which resulted to have a very low seismic rating, and thus a high priority for intervention planning.

\begin{tabular}{ccc} 
Element & DLS & LSLS \\
\hline Supports & $3.523 \mathrm{E}-04$ & $2.775 \mathrm{E}-02$ \\
Abutments & $0.000 \mathrm{E}+00$ & $1.898 \mathrm{E}-05$ \\
Piers & $9.948 \mathrm{E}-01$ & $1.832 \mathrm{E}-01$ \\
\hline Global & $9.948 \mathrm{E}-01$ & $2.059 \mathrm{E}-01$ \\
\hline
\end{tabular}

Table 5: Exceedance probability of structural components and of entire bridge and for case study. 


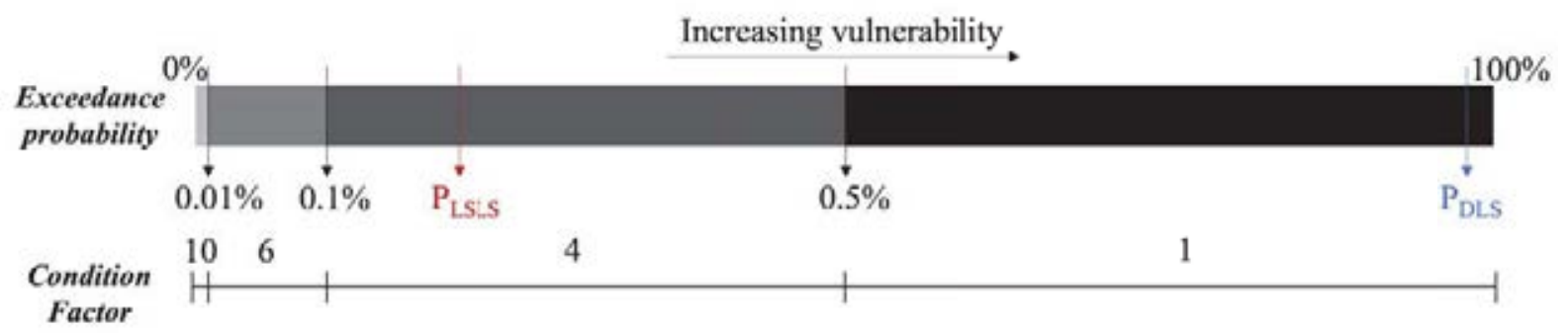

Figure 3: Condition factors associated to exceedance probability with values for case study for LSLS and DLS.

\begin{tabular}{cccc} 
& DLS & LSLS & Final \\
\hline TSR $_{\text {seismic }}$ & 9 & 36 & 9 \\
Seismic Class & Ds & Cs & Ds
\end{tabular}

Table 6: Evaluated seismic rating and class for case study.

\subsection{Application to the Padova bridge inventory}

The seismic-based ranking procedure was applied to 145 bridges of the Padova inventory belonging to macro-classes identified as prone to seismic damage.

The proposed procedure takes into account the local hazard through the demand PGA which value allows the probability of exceeding a LS to be obtained by using fragility curves. Since, in the current paper, the procedure was applied to an urban area with homogeneous seismic hazard classification (i.e., ground acceleration on soil A $a_{g} \simeq 0.1 g$ for relevant and strategic structures), no variability in the results is due to different hazard input, while it can be assumed to be linked to structural and typological characteristics.

Results are provided as follows in terms of seismic classes. Figure 4 reports the obtained seismic classes, for both bridges in the historical center and outside of it. The majority of structures in the $\mathrm{HC}$ are classified Cs (i.e., needing short-term retrofit interventions), while outside the $\mathrm{HC}$, most bridges (64\%) fall in the best two classes (As and Bs).

To better understand these distributions, Figure 5 provided the obtained seismic classes, disaggregated on the basis of construction age, deck material, and static scheme, for the entire stock (graphs) and for either HC or the areas outside it (tables).

Comparing the frequency of seismic classes for bridges inside and outside the $\mathrm{HC}$ (observing the distributions based on construction age, deck material, and static scheme) a generalized greater seismic vulnerability of the historical center network is confirmed. Higher frequencies are reported for class $\mathrm{Cs}$ in the $\mathrm{HC}$, and for the first two classes (As and Bs) outside the HC.

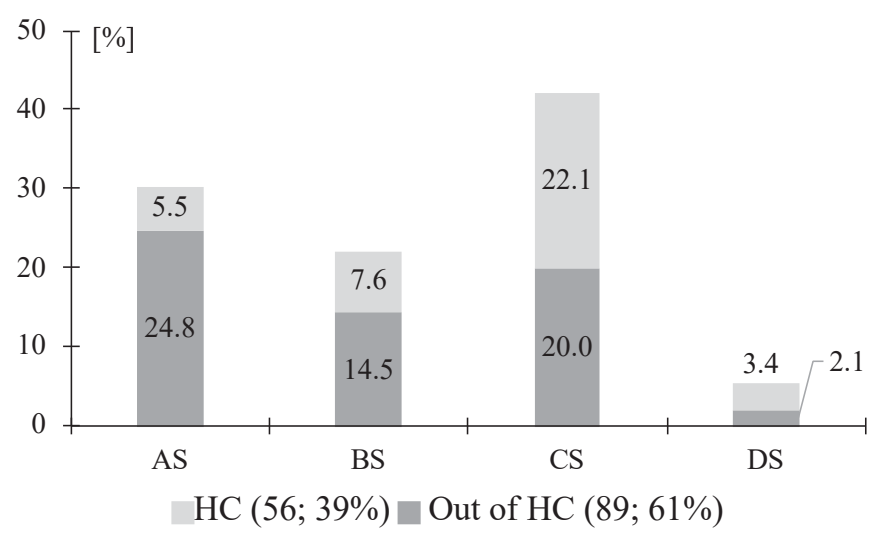

Figure 4: Distribution of seismic classes obtained for earthquake prone bridges of Padova inventory. 


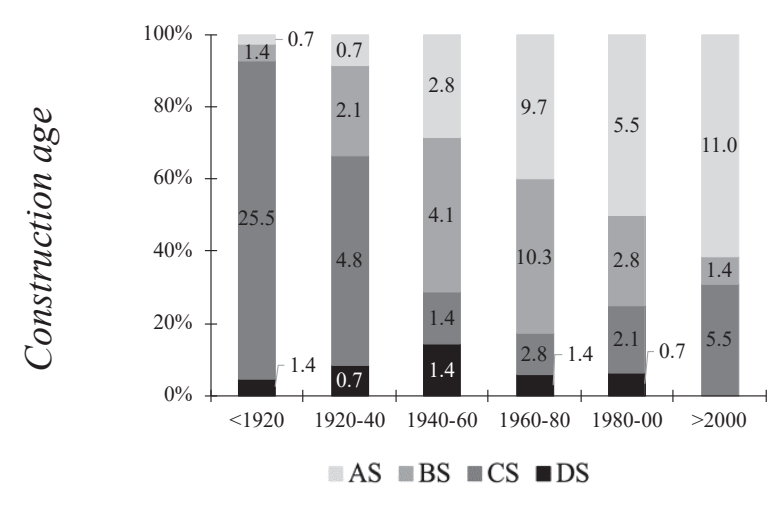

\begin{tabular}{|c|c|c|c|c|c|}
\hline \multicolumn{2}{|c|}{ HC } & \multicolumn{4}{|c|}{ Seismic classes } \\
\hline 56 & [\$] & A & B. & c & D. \\
\hline \multirow{6}{*}{ 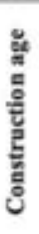 } & $<1920$ & 1.8 & 3.6 & 51.8 & 3.6 \\
\hline & $1920-40$ & 0 & 1.8 & 3.6 & 1.8 \\
\hline & $1940-60$ & 3.6 & 7.1 & 1.8 & 3.6 \\
\hline & $1960-80$ & 0 & 5.4 & 0 & 0 \\
\hline & $1980-00$ & 5.4 & 1.8 & 0 & $\mathbf{0}$ \\
\hline & $>2000$ & 3.6 & 0 & 0 & 0 \\
\hline
\end{tabular}

\begin{tabular}{|c|c|c|c|c|c|}
\hline \multicolumn{2}{|c|}{ Out of HC } & \multicolumn{4}{|c|}{ Selsmic classes } \\
\hline 89 & {$[\%]$} & A & B. & c. & D. \\
\hline \multirow{6}{*}{ 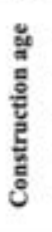 } & $<1920$ & 0 & 0 & 9.0 & 0 \\
\hline & $1920-40$ & 1.1 & 2.2 & 5.6 & 0 \\
\hline & $1940-60$ & 2.2 & 2.2 & 1.1 & 0 \\
\hline & $1960-80$ & 15.7 & 13.5 & 4.5 & 2.2 \\
\hline & $1980-00$ & 5.6 & 3.4 & 3,4 & 1.1 \\
\hline & $>2000$ & 15.7 & 2.2 & 9.0 & 0 \\
\hline
\end{tabular}

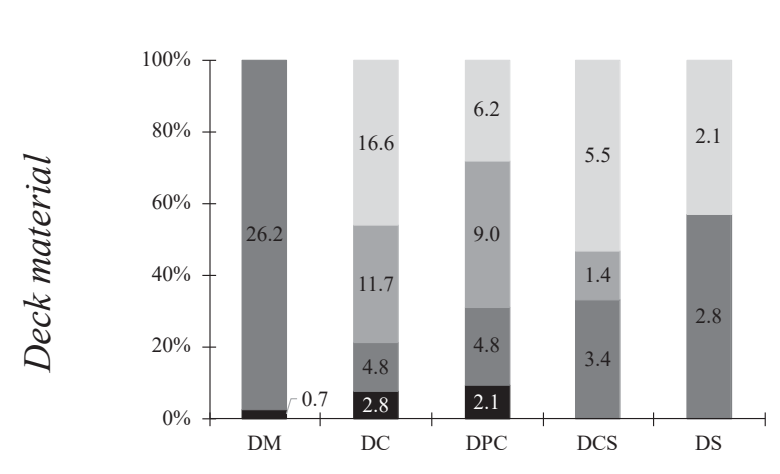

\begin{tabular}{|c|c|c|c|c|c|}
\hline \multicolumn{2}{|c|}{ HC } & \multicolumn{4}{|c|}{ Seismic classes } \\
\hline 56 & [N] & $A_{0}$ & B. & c & D \\
\hline \multirow{5}{*}{ 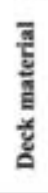 } & DM & 0 & 0 & 51.8 & 1.8 \\
\hline & DC & 12.5 & 17.9 & 5.4 & 7.1 \\
\hline & DPC & 0 & 0 & 0 & 0 \\
\hline & DCS & 1.8 & 1.8 & 0 & 0 \\
\hline & DS & 0 & 0 & 0 & 0 \\
\hline
\end{tabular}

\begin{tabular}{|c|c|c|c|c|c|}
\hline \multicolumn{2}{|c|}{ Out of HC } & \multicolumn{4}{|c|}{ Selsmie classes } \\
\hline 89 & [\%] & A & B. & c. & D. \\
\hline \multirow{5}{*}{ 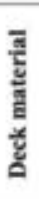 } & DM & 0 & 0 & 10.1 & 0 \\
\hline & DC & 19.1 & 7.9 & 4.5 & 0 \\
\hline & DPC & 10.1 & 14.6 & 7.9 & 3.4 \\
\hline & DCs & 7.9 & 1.1 & 5.6 & 0 \\
\hline & DS & 3.4 & 0 & 4.5 & 0 \\
\hline
\end{tabular}

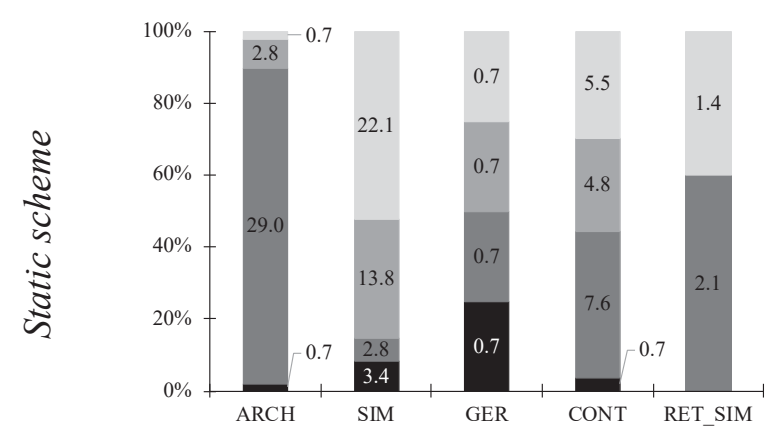

\begin{tabular}{|c|c|c|c|c|c|}
\hline \multicolumn{2}{|c|}{ HC } & \multicolumn{4}{|c|}{ Seismic classes } \\
\hline 56 & {$[\%]$} & $A_{0}$ & $\mathrm{~B}_{4}$ & c & D. \\
\hline & $A R C H$ & 0 & 5.4 & 57.1 & 1.8 \\
\hline & SMM & 125 & 5,4 & 0 & 3.6 \\
\hline & GER & 0 & 0 & 0 & 1.8 \\
\hline & CONT & 1.8 & 8.9 & 0 & 1.8 \\
\hline & RET_SIM & 0 & 0 & 0 & 0 \\
\hline
\end{tabular}

\begin{tabular}{|c|c|ccc|c|}
\hline \multicolumn{2}{|c|}{${ }^{2}$ HC } & \multicolumn{4}{|c|}{ Seismic classes } \\
\hline [\%] & A & B. & C & D. \\
\hline ARCI & 1.1 & 1.1 & 11.2 & 0 \\
\hline SIM & 28.1 & 19.1 & 4.5 & 3.4 \\
\hline GER & 1.1 & 1.1 & 1.1 & 0 \\
\hline CONT & 7.9 & 2.2 & 12.4 & 0 \\
\hline RET_SIM & 2.2 & 0 & 3.4 & 0 \\
\hline
\end{tabular}

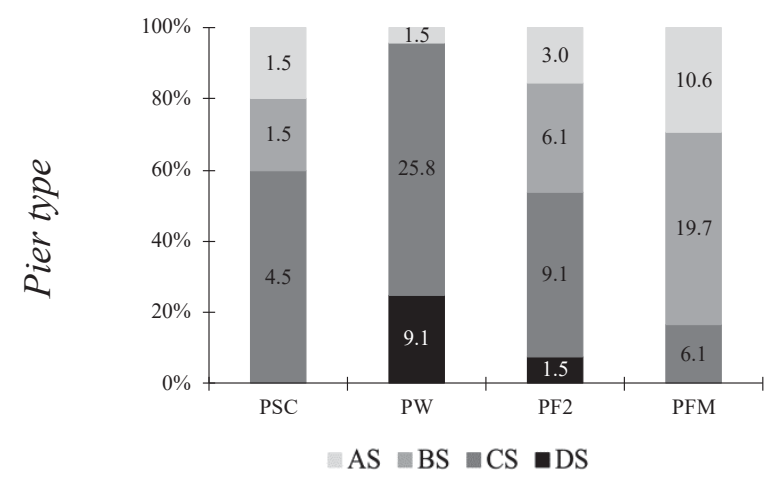

\begin{tabular}{|c|c|c|c|c|c|}
\hline \multicolumn{2}{|c|}{ HC } & \multicolumn{4}{|c|}{ Seismic classes } \\
\hline 22 & {$[\mathbf{*}]$} & $A_{t}$ & B. & c. & D. \\
\hline \multirow{4}{*}{$\frac{\mathrm{g}}{\mathrm{g}}$} & PSC & 0 & 0 & 0 & 0 \\
\hline & $p w$ & 0 & 0 & 45.5 & 18.2 \\
\hline & $P F 2$ & 0 & 4.5 & 0 & 0 \\
\hline & PFM & 9.1 & 22.7 & 0 & 0 \\
\hline
\end{tabular}

\begin{tabular}{|c|c|c|c|c|c|}
\hline \multicolumn{2}{|c|}{ Out of HC } & \multicolumn{4}{|c|}{ Selsmic classes } \\
\hline 4 & 104 & A. & B. & c. & D. \\
\hline \multirow{4}{*}{$\frac{\mathrm{z}}{\mathrm{z}}$} & $\mathrm{PSC}$ & 2.3 & 2.3 & 6.8 & 0 \\
\hline & $p \mathrm{w}$ & 2.3 & 0 & 15.9 & 4.5 \\
\hline & $m 2$ & 4.5 & 6.8 & 13.6 & 2.3 \\
\hline & $\mathrm{FM}$ & 11.4 & 18.2 & 9.1 & 0 \\
\hline
\end{tabular}

Figure 5: Obtained seismic classes for earthquake prone bridges of Padova inventory disaggregated on the basis of construction age, deck material, and static scheme. 
Focusing on the distribution of seismic classes during construction periods, as expected, a larger amount of As-classified structures is found as the age of bridges decreases. The vast majority of bridges built before 1920 falls in seismic class Cs, while various distributions are found in the intermediate ages. Bridges built in the decades 1940s-1960s present the larger portion of structures classified Ds (i.e., needing immediate planning for seismic retrofit interventions).

An interesting correlation emerges analyzing the distribution of seismic classes for different deck materials; most of masonry bridges are classified Cs (leading to the high percentage of structures classified Cs among bridges built before 1920 and with arch static scheme). The critical mechanism for the majority of these bridges was indeed the overturning of spandrel walls, leading to the same seismic class.

R.c. bridges (both ordinary and precast) report the highest percentage of Ds classified structures. The most vulnerable structures, in this case, presents squat wall piers, with high probability of exceeding the trigger of shear mechanism at Damage LS.

A limited number (22) of steel bridges was analyzed in this contribution, since various steel structures were excluded due to their lightness and flexibility. None of this type of structures is classified Ds. Although caution must be applied to the interpretation of results due to the small sample size, a possible explanation for this might be that steel bridges are, on average, of recent construction.

The application of seismic-based prioritization allows typological intervention to be identified, based on specific deficiencies which characterized some types of bridges. Retrofitting all the masonry arched bridges classified Cs by means of tie-rods, to prevent spandrel wall overturning, is a fast intervention, with low impact and cost and great efficiency. Indeed, it allows bridges to change seismic class from Cs to As, with great impact on the global vulnerability of the stock, requiring limited effort.

A common deficiency for girder bridges is given by inadequate support devices. To replace supports with more efficient isolation systems would allow seismic vulnerability of structures to be reduced, with also a decreasing in the forces transferred to substructures.

\section{COMPARISON WITH ITALIAN GUIDELINES}

Very recently, novel Italian Guidelines for the assessment of bridge stocks were issued [25]. The proposed multi-level approach includes a procedure to classify bridges in five "warning levels" (i.e., high, medium-high, medium, medium-low, low) [30]. The estimation of warning levels, indeed, represent "Level 2" of the guideline procedure, preceded by inventory of the analyzed asset and visual inspection, and followed by detailed evaluations and verifications.

The approach allows the combination, through logical operators, of warning levels referring to various natural hazards (i.e., seismic, hydraulic, and hydrogeological), as well as to degradation effects.

The evaluation of warning levels associated to seismic risk was carried out for the abovedescribed bridge inventory of Padova. In this section results from Italian Guidelines are presented and compared to the outcomes of the proposed seismic-based ranking procedure.

The main difference between the outcomes of these approaches is that the Guidelines group structures in warning levels, in which homogeneous risk is assumed, while the proposed ranking procedure provides a quantitative rating, allowing bridges to be sorted according to the estimated vulnerability.

The approach provided by the Guidelines combines, through logic paths, factors of hazard, exposure and vulnerability, which in turn depend on the following parameters: i) hazard parameters are the peak ground acceleration, topography, and type of soil; ii) exposure parameters are traffic volume, average span length and importance of the bridge; iii) vulnerability parameters 
are construction material, static scheme and span length, as well as degradation level and design criteria (whether seismic design was considered or not).

Figure 6 compares the distribution of seismic classes, obtained with the above-described seismic based ranking procedure, and the warning level toward seismic risk obtained from the application of Italian Guidelines [25]. First, the classification of all the seismic prone bridges of the Padova inventory are reported (Figure 6a). The guideline approach provides more conservative results, with most bridges which presents a high or medium-high warning level, and no structure with low warning level. For a local administration in charge with a relatively large stock of structure, it could be challenging to manage the outcomes of guideline prioritization, as the number of bridges requiring urgent verifications remains high. Moreover, when an extensive retrofit campaign must be planes, allocating limited funds, the identification of a manageable number of most critical structures is necessary.

Results of the different approaches are compared also on the basis of deck material (Figure 6b), construction age (Figure 6c), and static scheme (Figure 6d).

Generally, results from the guideline approach are confirmed to be more conservative; an exception is given by masonry arche bridges, which classification according to Guidelines is quite heterogeneous, while they were classified mainly Cs by the proposed seismic-based prioritization. This result may be explained by the fact that the evaluation of warning level according to Italian Guidelines is focused on the Life-Safety LS of the structure, thus some damage mechanisms involving secondary elements (e.g., spandrel walls in masonry arch bridges) may be not directly included. On the contrary, the proposed seismic-based approach includes this kind of mechanisms, which, in case of masonry arch bridges, results to be the most critical, affecting the seismic classification. R.c. bridges report the highest percentage of the worst classified structures, for both the approaches.

The distribution of the most vulnerable structure, according to the analyzed procedure, across the age of construction is slightly different, with a concentration of high warning level [25] in the period 1960-1980, while, as mentioned before, bridges built in the preceding decades (1940-1960) present the peak of Ds-classified structures.

The guideline procedure results to be punitive in particular for bridges with simply supported deck (both simply supported girder and Gerber scheme deck), which however present a peak of worst classified structures also by applying the proposed seismic based ranking procedure.

\section{CONCLUSIONS}

- This paper discusses a priority ranking procedure presented by the authors in Saler et al. [26]. Since 2016, the prioritization approach has been applied to an urban bridge stock managed by the Municipality of Padova, in North-East Italy.

- An extensive campaign of visual inspection was carried out, allowing a detailed taxonomy of the urban stock to be devised and presented in the current contribution by means of aggregated distributions of structural and typological characteristics. Data related to the historical center have been unbundled, so as to highlight its particular features.

- The evaluation of the seismic vulnerability of the stock through fragility curves for various mechanisms allows some indications about typological interventions to be deduced: i) masonry arched bridges are mainly subjected to spandrel wall overturning, thus the application of tie-rods (with low cost and impact) is suitable to improve the seismic response of this type of bridges; ii) girder bridges often have inadequate support systems which replacement with isolation devices can reduce the seismic vulnerability of both substructures and superstructures. 

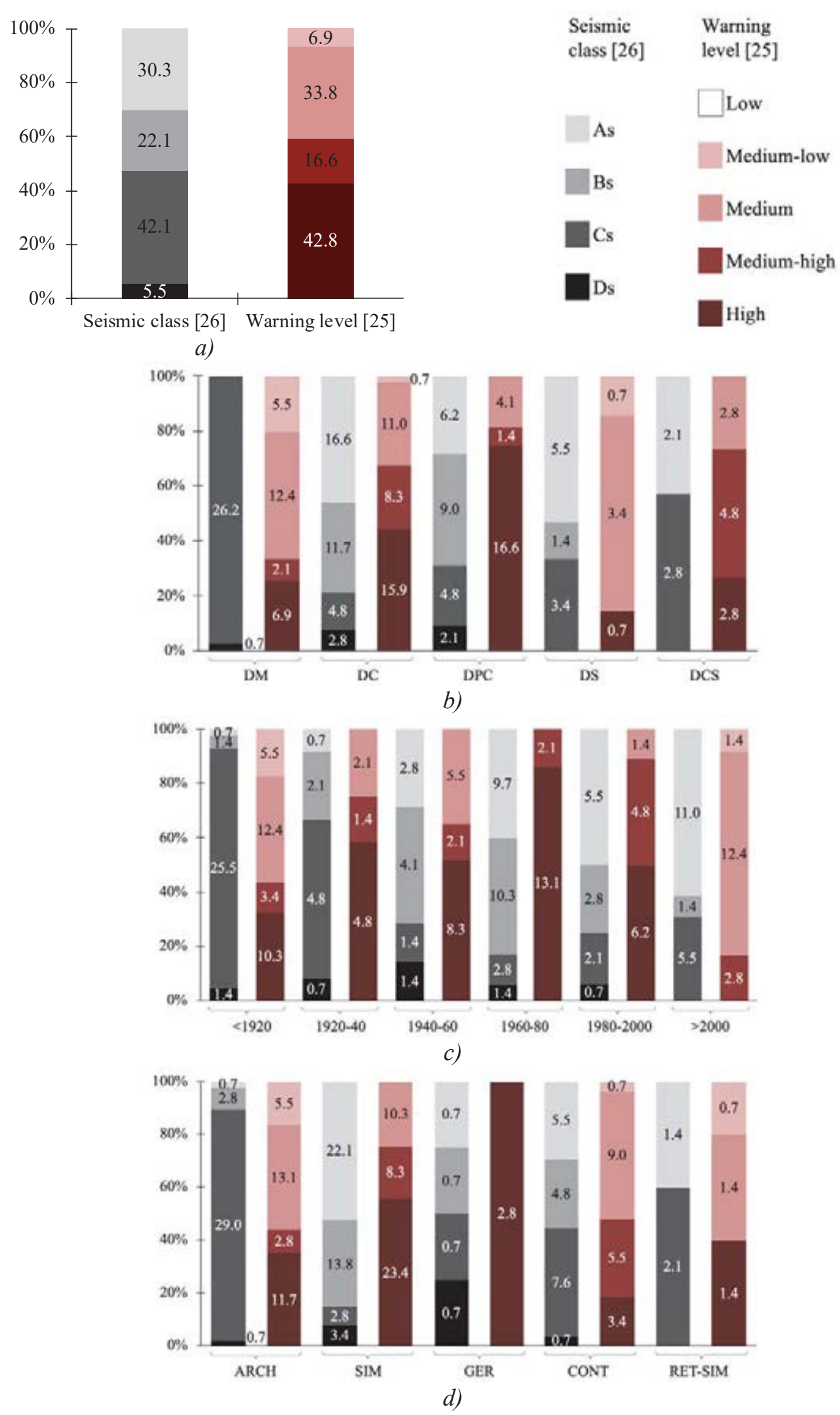

Figure 6: Comparison of seismic class [26] and warning level [25] distributions: a) general; b) based on deck material; c) based on construction age; and d) based on static scheme. 
- The evaluation of seismic warning levels according to novel Italian Guidelines on existing bridges [25] was carried out for the bridge inventory of Padova. Italian Guidelines group structures in warning levels (homogeneous risk), while the proposed approach rates each structure, allowing bridges to be sorted according to their vulnerability.

- Results from the applied procedures have been compared. Guidelines resulted to be more conservative, with most bridges presenting a high or medium-high warning level, and no structure with low warning level. However, similar results might be difficult to be managed by local administrations in charge, for the high number of bridges requiring urgent verifications to be urgently planned.

\section{ACKNOWLEDGEMENTS}

Special thanks are due to the Municipality of Padova which partly funded this work.

\section{REFERENCES}

[1] A. Del Grosso, D. Inaudi, L. Pardi, Overview of European activities in the health monitoring of bridges, First Int. Conf. Bridg. Maintenance, Saf. Manag., 2002 1-8.

[2] G. Grunthal, EMS98 - European Macroseismic Scale 1998, Conseil de l'Europe - Cahiers du Centre Européen de Géodynamique et de Séismologie, Luxemburg, 1998.

[3] A.H.M. Muntasir Billah, M. Shahria Alam, Seismic fragility assessment of highway bridges: a state-of-the-art review, Struct. Infrastruct. Eng. 11, 2015, 804-832. https://doi.org/10.1080/15732479.2014.912243.

[4] E. Choi, R. DesRoches, B. Nielson, Seismic fragility of typical bridges in moderate seismic zones, Eng. Struct. 26, 2004, 187-199. https://doi.org/10.1016/j.engstruct.2003.09.006.

[5] Y. Pan, A.K. Agrawal, M. Ghosn, S. Alampalli, Seismic fragility of multispan simply supported steel highway bridges in New York State. II: Fragility analysis, fragility curves, and fragility surfaces, J. Bridg. Eng. 15, 2010, 462-472. https://doi.org/10.1061/(ASCE)BE.1943-5592.0000055.

[6] D.H. Tavares, J.E. Padgett, P. Paultre, Fragility curves of typical as-built highway bridges in eastern Canada, Eng. Struct. 40, 2012, 107-118. https://doi.org/10.1016/j.engstruct.2012.02.019.

[7] J. Ghosh, J.E. Padgett, Aging considerations in the development of time-dependent seismic fragility curves, J. Struct. Eng. 136, 2010, 1497-1511. https://doi.org/10.1061/(ASCE)ST.1943-541X.0000260.

[8] G. Tecchio, Simplified displacement-based approaches for seismic design and vulnerability assessment of mulit-span rc bridges, PhD Thesis, University of Padova, 2013.

[9] G. Tecchio, M. Donà, C. Modena, Direct displacement-based design accuracy prediction for single-column RC bridge bents, Earthq. Struct. 9, 2015, 455-480. https://doi.org/10.12989/eas.2015.9.3.455.

[10] F. da Porto, G. Tecchio, P. Zampieri, C. Modena, A. Prota, Simplified seismic 
assessment of railway masonry arch bridges by limit analysis, Struct. Infrastruct. Eng. 12, 2015, 567-591. https://doi.org/10.1080/15732479.2015.1031141.

[11] G. Tecchio, M. Donà, F. da Porto, Seismic fragility curves of as-built single-span masonry arch bridges, Bull. Earthq. Eng. 14, 2016, 3099-3124. https://doi.org/10.1007/s10518-016-9931-6.

[12] D. Cardone, G. Perrone, M. Dolce, a Numerical Procedure for the Assessment of Highway Bridges in Seismic Area, in: Proc. ECCOMAS Themat. Conf. Comput. Methods Struct. Dyn. Earthq. Eng. (COMPDYN, 2007) Rethymno, Crete, Greece, 2007: pp. 1316.

[13] P.E. Pinto, P. Franchin, Issues in the upgrade of Italian highway structures, J. Earthq. Eng., 14:8, 1221-1252, 2010. https://doi.org/10.1080/13632461003649970.

[14] B. Borzi, P. Ceresa, P. Franchin, F. Noto, G.M. Calvi, P.E. Pinto, Seismic vulnerability of the Italian roadway bridge stock, Earthq. Spectra. 31, 2015, 2137-2161. https://doi.org/10.1193/070413EQS190M.

[15] L. Deng, W. Wang, Y. Yu, State-of-The-Art Review on the Causes and Mechanisms of Bridge Collapse, J. Perform. Constr. Facil. 30, 2016, 1-13. https://doi.org/10.1061/(ASCE)CF.1943-5509.0000731.

[16] G. Tecchio, F. Lorenzoni, M. Caldon, M. Donà, F. da Porto, C. Modena, Monitoring of orthotropic steel decks for experimental evaluation of residual fatigue life, J. Civ. Struct. Heal. Monit. 7, 2017, 517-539. https://doi.org/10.1007/s13349-017-0240-9.

[17] F. Lorenzoni, N. De Conto, F. da Porto, C. Modena, Ambient and free-vibration tests to improve the quantification and estimation of modal parameters in existing bridges, J. Civ. Struct. Heal. Monit. 9, 2019, 617-637. https://doi.org/10.1007/s13349-019-00357-4.

[18] P.D. Thompson, E.P. Small, M. Johnson, A.R. Marshall, The Pontis Bridge Management System, Struct. Eng. Int. J. Int. Assoc. Bridg. Struct. Eng., 1998,. https://doi.org/10.2749/101686698780488758.

[19] H. Hawk, E.P. Small, The BRIDGIT Bridge Management System, Struct. Eng. Int. J. Int. Assoc. Bridg. Struct. Eng. 8, 1998. https://doi.org/10.2749/101686698780488712.

[20] R.J. Woodward, C. D.W., A.F. Daly, P.R. Vassie, P. Haardt, R. Kashner, R. Astudillo, C. Velando, B. Godart, C. Cremona, B. Mahut, A. Raharinaivo, Lau, I. Markey, L. Bevc, I. Peruš, BRIME - Bridge Management in Europe, Final Rep. (2001) 227.

[21] V. Gattulli, L. Chiaramonte, Condition assesment by visual inspection for a bridge management system, Comput. Civ. Infrastruct. Eng. 20, 2005, 95-107. https://doi.org/10.1111/j.1467-8667.2005.00379.x.

[22] A. Montepara, F. Merusi, F. Giuliani, Sviluppo di una nuova metodologia per la valutazione delle priorità di intervento di manutenzione di ponti e viadotti, $17^{\circ}$ Convegno Naz. Della Soc. Ital. Infrastrutture Viarie. (2008).

[23] S. Valenzuela, H. De Solminihac, T. Echaveguren, Proposal of an integrated index for prioritization of bridge maintenance, J. Bridg. Eng. 15, 2010, 337-343. https://doi.org/10.1061/(ASCE)BE.1943-5592.0000068.

[24] D.Y. Yang, D.M. Frangopol, Risk-informed bridge ranking at project and network levels, J. Infrastruct. Syst. 24, 2018. https://doi.org/10.1061/(ASCE)IS.1943-555X.0000430. 
[25] C.S.LL.PP, Linee Guida per la classificazione e gestione del rischio, la valutazione della sicurezza ed il monitoraggio di ponti esistenti (in Italian), 2020.

[26] E. Saler, V. Pernechele, M. Anastasio, M. Minotto, G. Tecchio, Macroscale vulnerability assessment of bridges at municipality level, in: Atti Del XVIII Convegno ANIDIS L'ingegneria Sismica Ital. Ascoli Piceno, 15-19 Settembre 2019, 2019.

[27] C. Pellegrino, A. Pipinato, C. Modena, A simplified management procedure for bridge network maintenance, Struct. Infrastruct. Eng. 7, 2011, 341-351. https://doi.org/10.1080/15732470802659084.

[28] Italian Department of Civil Protection, Clarifications on the management of the results of seismic verifications carried out in compliance with OPCM 3274/2003 (in Italian), (2010). http://www.protezionecivile.gov.it/documents/20182/0/Gestioneverifiche.pdf/c6870ced-e049-494c-84de-2689d83b993c.

[29] OPCM 3274/2003, All. II - Technical standards for the project, the assessment and the seismic retrofit of buildings (in Italian), (2003).

[30] F. Braga, G. Buratti, A. Cosentino, A. Dall, G. De Matteis, Multi-level approach for the assessment of bridge and viaducts within road networks, in: Atti Del XVIII Convegno ANIDIS L’ingegneria Sismica Ital. Ascoli Piceno, 15-19 Settembre 2019, 2019. 